\title{
Feedback Stabilization Methods For The Numerical Solution Of Systems Of Ordinary Differential Equations
}

\author{
Iasson Karafyllis ${ }^{*}$ and Lars Grüne** \\ "Department of Environmental Engineering, \\ Technical University of Crete,73100, Chania, Greece \\ email: ikarafyl@enveng.tuc.gr \\ ** Mathematisches Institut, Fakultät für Mathematik und Physik, \\ Universität Bayreuth, 95440 Bayreuth, Germany \\ email: lars.gruene@uni-bayreuth.de
}

\begin{abstract}
In this work we study the problem of step size selection for numerical schemes, which guarantees that the numerical solution presents the same qualitative behavior as the original system of ordinary differential equations, by means of tools from nonlinear control theory. Lyapunov-based stabilization methods are exploited.
\end{abstract}

Keywords: Stability of numerical schemes, feedback stabilization, nonlinear systems.

PACS: $02.60 . \mathrm{Cb}, 02.30 . \mathrm{Hq}$

\section{INTRODUCTION}

It is well-known that step size control can enhance the performance of a numerical scheme when applied to a system of Ordinary Differential Equations (ODEs). For example, in [2] the authors use a "Proportional-Integral" technique which is similar to the "Proportional-Integral" controller used in Linear Systems Theory in order to keep the local discretization error within certain bounds (see also [3,4,8]). Theoretical results on the behavior of adaptive time-stepping methods have been presented in [14,15].

In this work, we develop tools for nonlinear systems which are similar to methods used in Nonlinear Control Theory. We consider the problem of selecting the step size for numerical schemes so that the numerical solution presents the same qualitative behavior as the original system of ODEs. It is well-known that any consistent and stable numerical scheme for ODEs inherits the asymptotic stability of the original equation in a practical sense, see for instance [5,6] and [16] (Chapter 7). In contrast to these results, in this paper we investigate the case in which the numerical approximation is asymptotically stable in the usual sense, i.e., not only practically. Here, we concentrate on nonlinear systems for which an equilibrium point is the global attractor. In the following, it is shown how the problem of appropriate step size selection can be converted to a rigorous abstract feedback stabilization problem for a particular hybrid system (see also [11]-the reader should notice that the standard stability analysis of numerical schemes uses the study of a discrete-time system e.g., [8,9,10,16]; not a hybrid system). Therefore, we are in a position to use all methods of feedback design for nonlinear systems. Specifically, we consider methods based on Small-Gain Theorems and methods based on Lyapunov functions. Both methods have been used widely in Nonlinear Systems Theory for the solution of feedback stabilization problems (see [1,13] and references therein). However, in the present work, due to space limitations we consider only Lyapunov-based methods for the step size selection for numerical schemes for ODEs (see [12] for more details). General results are developed for arbitrary consistent Runge-Kutta schemes (see Theorem 3 below) and specific results are given for specific Runge-Kutta schemes (see Theorem 4 below). Due to space limitations all proofs are omitted and can be provided upon request of the authors. The obtained results have numerous applications (e.g., application of explicit schemes for stiff problems) which are presented in [12]. 
Notations Let $A \subseteq \mathfrak{R}^{n}$ be a set. By $C^{0}(A ; \Omega)$, we denote the class of continuous functions on $A$, which take values in $\Omega$. By $C^{k}(A ; \Omega)$, where $k \geq 1$ is an integer, we denote the class of functions on $A$ with continuous derivatives up to order $k$, which take values in $\Omega$. For a vector $x \in \mathfrak{R}^{n}$ we denote by $|x|$ its usual Euclidean norm. $\mathfrak{R}^{+}$denotes the set of non-negative real numbers. For the classes of functions $K_{\infty}$ and $K L$ we adopt the definitions in [13].

\section{DESCRIPTION OF THE PROBLEM}

Consider the autonomous system

$$
\dot{z}(t)=f(z(t)), z(t) \in \mathfrak{R}^{n}
$$

where $f: \Re^{n} \rightarrow \Re^{n}$ is a locally Lipschitz vector field with $f(0)=0$. For every $z_{0} \in \mathfrak{R}^{n}$ and $t \geq 0$, the solution of (1) with initial condition $z(0)=z_{0}$ will be denoted by $z\left(t, z_{0}\right)$. The numerical approximation of system (1) will be the hybrid system:

$$
\begin{aligned}
& \dot{x}(t)=F\left(h_{i}, x\left(\tau_{i}\right)\right), t \in\left[\tau_{i}, \tau_{i+1}\right) \\
& \tau_{0}=0, \tau_{i+1}=\tau_{i}+h_{i}, h_{i}=\varphi\left(x\left(\tau_{i}\right)\right) \exp \left(-u\left(\tau_{i}\right)\right) \quad, \quad x(t) \in \mathfrak{R}^{n}, u(t) \in[0,+\infty)
\end{aligned}
$$

where $\varphi \in C^{0}\left(\Re^{n} ;(0, r]\right), r>0$ is a constant, $F: \underset{x \in \mathfrak{R}^{n}}{\cup}([0, \varphi(x)] \times\{x\}) \rightarrow \Re^{n}$ is a (not necessarily continuous) vector field with $F(h, 0)=0$ for all $h \in[0, \varphi(0)], \lim _{h \rightarrow 0^{+}} F(h, z)=f(z)$, for all $z \in \mathfrak{R}^{n}$. More specifically, the solution $x(t)$ of the hybrid system (2) is obtained for every locally bounded $u: \Re^{+} \rightarrow \Re^{+}$and $x_{0} \in \Re^{n}$ by the following algorithm (see [11]):

Step $i$ :

1) Given $\tau_{i}$ and $x\left(\tau_{i}\right)$, calculate $\tau_{i+1}$ using the equation $\tau_{i+1}=\tau_{i}+\varphi\left(x\left(\tau_{i}\right)\right) \exp \left(-u\left(\tau_{i}\right)\right)$,

2) Compute the state trajectory $x(t), t \in\left(\tau_{i}, \tau_{i+1}\right]$ as the solution of the differential equation $\dot{x}(t)=F\left(h_{i}, x\left(\tau_{i}\right)\right)$, i.e., $x(t)=x\left(\tau_{i}\right)+\left(t-\tau_{i}\right) F\left(h_{i}, x\left(\tau_{i}\right)\right)$ for $t \in\left(\tau_{i}, \tau_{i+1}\right]$.

For $i=0$ we take $\tau_{0}=0$ and $x(0)=x_{0}$ (initial condition). We will further assume that there exists a continuous, non-decreasing function $M: \mathfrak{R}^{+} \rightarrow \mathfrak{R}^{+}$such that

$$
|F(h, x)| \leq|x| M(|x|) \text { for all } x \in \mathfrak{R}^{n} \text { and } h \in[0, \varphi(x)]
$$

It should be noticed that the hybrid system (2) under hypothesis (3) is an autonomous system, which satisfies the "Boundedness-Implies-Continuation” property and for each locally bounded input $u: \mathfrak{R}^{+} \rightarrow \mathfrak{R}^{+}$and $x_{0} \in \mathfrak{R}^{n}$ there exists a unique absolutely continuous function $[0,+\infty) \ni t \rightarrow x(t) \in \mathfrak{R}^{n}$ with $x(0)=x_{0}$ which satisfies (2) (see [11]). All consistent $s$-stage Runge-Kutta methods can be represented by the hybrid system (2). More specifically, let $x_{0} \in \mathfrak{R}^{n}$ and consider a consistent $s$-stage Runge-Kutta method for (1):

We define

$$
Y_{i}=x_{0}+h \sum_{j=1}^{s} a_{i j} f\left(Y_{j}\right) \quad, \quad i=1, \ldots, s ; \quad x=x_{0}+h \sum_{i=1}^{s} b_{i} f\left(Y_{i}\right), \quad \sum_{i=1}^{s} b_{i}=1
$$

$$
F\left(h, x_{0}\right):=h^{-1}\left(x-x_{0}\right)
$$

A moment's thought reveals that for every locally bounded $u: \Re^{+} \rightarrow \mathfrak{R}^{+}$and $x_{0} \in \mathfrak{R}^{n}$ the solution of (2) with (5) coincides at each $\tau_{i}, i \geq 0$ with the numerical solution of (1) with $x(0)=x_{0}$ obtained by using the Runge-Kutta numerical scheme (4) and using the discretization step sizes $h_{i}=\varphi\left(x\left(\tau_{i}\right)\right) \exp \left(-u\left(\tau_{i}\right)\right), i \geq 0$. If the Runge-Kutta scheme (4) is of order $p \geq 1$, we will occasionally further assume that $f \in C^{p}\left(\Re^{n} ; \Re^{n}\right)$ and for each fixed $x \in \Re^{n}$ the mapping $[0, \varphi(x)] \ni h \rightarrow F(h, x)$ is $p$ times continuously differentiable with 


$$
|F(h, x)|+\sum_{j=1}^{p}\left|\frac{\partial^{j}}{\partial h^{j}} F(h, x)\right| \leq G(|x|) \max \left\{|f(y)|: y \in \mathfrak{R}^{n},|y-x| \leq|x| \varphi(x) M(|x|)\right\} \text { for all } x \in \mathfrak{R}^{n} \text { and } h \in[0, \varphi(x)]
$$

for some continuous, non-decreasing function $G: \mathfrak{R}^{+} \rightarrow \mathfrak{R}^{+}$, where $M: \mathfrak{R}^{+} \rightarrow \mathfrak{R}^{+}$is the function involved in (3). Using Theorem II.3.1 in [7], we may conclude that there exist continuous functions $N: \Re^{n} \rightarrow(0,+\infty)$, $C: \mathfrak{R}^{n} \rightarrow \mathfrak{R}^{+}$such that the following inequalities hold for all $x \in \mathfrak{R}^{n}$ and $h \in[0, \varphi(x)]$ :

$$
\begin{gathered}
\left.C(x) \leq N(x) \mid \max \left\{|f(y)|: y \in \mathfrak{R}^{n},|y-x| \leq|x| \varphi(x) M(|x|)\right\}+\max \{|f(z(h, x))|: h \in[0, \varphi(x)]\}\right] \\
|z(h, x)-x-h F(h, x)| \leq h^{p+1} C(x)
\end{gathered}
$$

Assume next that $0 \in \mathfrak{R}^{n}$ is Uniformly Globally Asymptotically Stable (UGAS) for (1). We would like to be in a position to know a continuous function $\varphi: \Re^{n} \rightarrow(0, r]$ so that the numerical solution produced by (2) has the correct qualitative behavior (e.g., $\lim _{t \rightarrow+\infty} x(t)=0$ ). However, we would like to be able to guarantee that the correct behavior for the numerical solution can be obtained by using arbitrary step sizes smaller than $\varphi\left(x\left(\tau_{i}\right)\right.$ ) (i.e., if we obtain the correct qualitative behavior using step sizes $h_{i}=\varphi\left(x\left(\tau_{i}\right)\right) \quad i \geq 0$, we would like to obtain the correct qualitative behavior using step sizes $h_{i}=\varphi\left(x\left(\tau_{i}\right)\right) \exp \left(-u\left(\tau_{i}\right)\right), i \geq 0$, where $u: \mathfrak{R}^{+} \rightarrow \mathfrak{R}^{+}$is an arbitrary locally bounded function). This is equivalent by requiring that $0 \in \mathfrak{R}^{n}$ is Uniformly Robustly Globally Asymptotically Stable (URGAS) for (2) (in the sense described in [11]). The reader should notice that continuity for the function $\varphi: \Re^{n} \rightarrow(0, r]$ is essential: without assuming continuity it may happen that $\liminf _{x \rightarrow 0} \varphi(x)=0$ and this would require step sizes of vanishing magnitude as $t \rightarrow+\infty$. Moreover, since we want to be able to determine a continuous function $\varphi: \Re^{n} \rightarrow(0, r]$, which "stabilizes" the hybrid system (2), we are essentially studying a feedback stabilization problem for the hybrid system (2). We consider the following feedback stabilization problems:

(P1)-Existence Problem: Is there a continuous function $\varphi: \mathfrak{R}^{n} \rightarrow(0, r]$, such that $0 \in \mathfrak{R}^{n}$ is URGAS for system (2)?

(P2)-Design Problem: Construct a continuous function $\varphi: \Re^{n} \rightarrow(0, r]$, such that $0 \in \mathfrak{R}^{n}$ is URGAS for system (2).

\section{LYAPUNOV FUNCTION BASED STEP SELECTION}

In this section we apply the Lyapunov-based feedback design methodology for the solution of Problems (P1) and (P2). It is well known that Lyapunov functions exist for every asymptotically stable ODE system. In the sequel, we will use a Lyapunov function for the continuous-time system in order to construct a Lyapunov function for its hybrid numerical approximation. To this end we use the following definition.

Definition 1: A positive definite, radially unbounded function $V \in C^{1}\left(\Re^{n} ; \Re^{+}\right)$is called a Lyapunov function for system (1) if $\nabla V(x) f(x)<0$ holds for all $x \in\left(\Re^{n} \backslash\{0\}\right)$.

In what follows, we show that under certain assumptions a Lyapunov function $V$ for (1) can be used as a Control Lyapunov Function (see [1]) in order to design the step function $\varphi: \Re^{n} \rightarrow(0, r]$ involved in problems (P1) and (P2). The following theorem provides sufficient conditions for the solvability of problem (P2) based on the Lyapunov function for the dynamical system (1).

Theorem 2: Consider system (2) that corresponds to a Runge-Kutta scheme for (1) of order $p \geq 1$ satisfying (6), (7), (8) for certain $\varphi \in C^{0}\left(\Re^{n} ;(0,+\infty)\right)$. Suppose that:

i) There exist a Lyapunov function $V \in C^{1}\left(\Re^{n} ; \Re^{+}\right)$for system (1) and a continuous, positive definite function $\tilde{W}: \Re^{n} \rightarrow \Re^{+}$such that the inequality $V(z(h, x)) \leq V(x)-h \tilde{W}(x)$ holds for all $x \in \Re^{n}$ and $h \in[0, \varphi(x)]$.

ii) There exists $b \geq 0$ such that $|z(h, x)| \leq \exp (b)|x|$ and $|x+h F(h, x)| \leq \exp (b)|x|$ for all $x \in \mathfrak{R}^{n}$ and $h \in[0, \varphi(x)]$. 
iii) There exists a constant $\lambda \in(0,1)$ such that

$$
\varphi(x) \leq\left(\frac{(1-\lambda) \tilde{W}(x)}{l_{V}^{b}(x) C(x)}\right)^{\frac{1}{p}}, \forall x \in\left(\Re^{n} \backslash\{0\}\right)
$$

where $l_{V}^{b}(x):=\max \left\{|\nabla V(z)|: z \in \mathfrak{R}^{n},|z| \leq \exp (b)|x|\right\}$ for all $x \in \mathfrak{R}^{n}$ and $C: \mathfrak{R}^{n} \rightarrow \mathfrak{R}^{+}$is a continuous positive definite function with $|z(h, x)-x-h F(h, x)| \leq C(x) h^{p+1}$ for all $x \in \mathfrak{R}^{n}$ and $h \in[0, \varphi(x)]$. Then $0 \in \mathfrak{R}^{n}$ is URGAS for system (2).

Based on Theorem 2, the following theorem shows that for the special case of a locally exponentially stable ODE system, problem (P1) is always solvable.

Theorem 3: Consider system (1) and a consistent Runge-Kutta scheme with order $p \geq 1$ and $f \in C^{p}\left(\Re^{n} ; \mathfrak{R}^{n}\right)$. Assume that $0 \in \mathfrak{R}^{n}$ is GAS and locally exponentially stable for (1). Then Problem (P1) is solvable.

Next, we show how Lyapunov function based arguments can be used for implicit schemes. It is clear that Theorem 4 generalizes the A-stability property to nonlinear systems.

Theorem 4-Implicit Euler Method: Suppose that there exists a convex Lyapunov function for (1), where $f \in C^{0}\left(\Re^{n} ; \Re^{n}\right)$ is simply locally Lipschitz. Let $\bar{\varphi} \in C^{0}\left(\Re^{n} ;(0,+\infty)\right)$ be such that the equation $Y=x+h f(Y)$ has a unique solution $Y \in \mathfrak{R}^{n}$ for all $h \in[0, \bar{\varphi}(x)]$ and $x \in \mathfrak{R}^{n}$. Then for each $r>0,0 \in \mathfrak{R}^{n}$ is URGAS for the corresponding system (2) with $F(h, x):=f(Y), \varphi(x):=\min \{\bar{\varphi}(x), r\}$, where $Y=x+h f(Y)$.

\section{REFERENCES}

1. Freeman, R. A. and P. V. Kokotovic, Robust Nonlinear Control Design- State Space and Lyapunov Techniques, Birkhauser, Boston, 1996.

2. Gustafsson, K., M. Lundh and G. Soderlind, "A PI Stepsize Control for the Numerical Solution of Ordinary Differential Equations”, BIT, 28, 1988, 270-287.

3. Gustafsson, K., "Control Theoretic Techniques for Stepsize Selection in Explicit Runge-Kutta Methods”, ACM Trans. Math. Soft., 17, 1991, 533-554.

4. Gustafsson, K., "Control-Theoretic Techniques for Stepsize Selection in Implicit Runge-Kutta Methods”, ACM Trans. Math. Soft., 20, 1994, 496-517.

5. Grüne, L., Asymptotic Behavior of Dynamical and Control Systems under Perturbation and Discretization, Springer-Verlag, 2002.

6. Grüne, L., “Attraction rates, robustness and discretization of attractors”, SIAM Journal on Numerical Analysis, 41, 2003, 20962113.

7. Hairer, E., S. P. Norsett and G. Wanner, Solving Ordinary Differential Equations I Nonstiff Problems, $2^{\text {nd }}$ Ed., SpringerVerlag, Berlin-Heidelberg, 1993.

8. Hairer, E. and G. Wanner, Solving Ordinary Differential Equations II Stiff and Differential-Algebraic Problems, $2^{\text {nd }}$ Edition, Springer-Verlag, Berlin-Heidelberg, 2002.

9. Hairer, E., C. Lubich and G. Wanner, Geometric Numerical Integration Structure Preserving Algorithms for Ordinary Differential Equations, Springer, 2002.

10. Karafyllis, I., "Non-Uniform Robust Global Asymptotic Stability for Discrete-Time Systems and Applications to Numerical Analysis”, IMA Journal of Mathematical Control and Information, 23(1), 2006, 11-41.

11. Karafyllis, I., “A System-Theoretic Framework for a Wide Class of Systems I: Applications to Numerical Analysis”, Journal of Mathematical Analysis and Applications, 328(2), 2007, 876-899.

12. Karafyllis, I. and L. Grüne, "Feedback Stabilization Methods For The Numerical Solution Of Systems Of Ordinary Differential Equations", submitted to BIT.

13. Khalil, H. K., Nonlinear Systems, $2^{\text {nd }}$ Edition, Prentice-Hall, 1996.

14. Kloeden, P.E. and J. Lorenz, "Stable Attracting Sets in Dynamical Systems and their One-Step Discretizations", SIAM Journal on Numerical Analysis, 23, 1986, 986-995.

15. Lamba, H., "Dynamical Systems and Adaptive Timestepping in ODE Solvers”, BIT Numerical Mathematics, 40(2), 2000, 314-335.

16. Stuart, A.M. and A.R. Humphries, Dynamical Systems and Numerical Analysis, Cambridge University Press, 1998. 